\title{
Chloride Deposits on Mars: Chlorine from the Sky, or Chlorine from the Rocks?
}

Mohit Melwani Daswani

Edwin S. Kite

Session 102, \# 3

GSA Denver - 26 ${ }^{\text {th }}$ September 2016 


\section{Overview/Problem}

Chloride-rich deposits are abundant on the highlands of Mars

...But their origin is unconstrained

...Source of $\mathrm{Cl}$ ? Environmental context? 


\section{Deposits can be quantified}

$1225 \mathrm{~km}^{2}$ basin

Chloride deposit:

$4 \mathrm{~m}$ max thickness

$29.83 \mathrm{~km}^{2}$

$\sim 0.12 \mathrm{~km}^{3}$

$\sim 1.4 \times 10^{11} \mathrm{~kg} \mathrm{NaCl}$

(assuming $45 \%$ porosity)

Lake:

$35.87 \mathrm{~km}^{3}$

$35.87 \times 10^{12} \mathrm{~kg} \mathrm{H}_{2} \mathrm{O}$

Salinity:

$\sim 4 \mathrm{~g} \mathrm{NaCl} / \mathrm{kg} \mathrm{H}_{2} \mathrm{O}$

Hynek et al. (2015) Geology
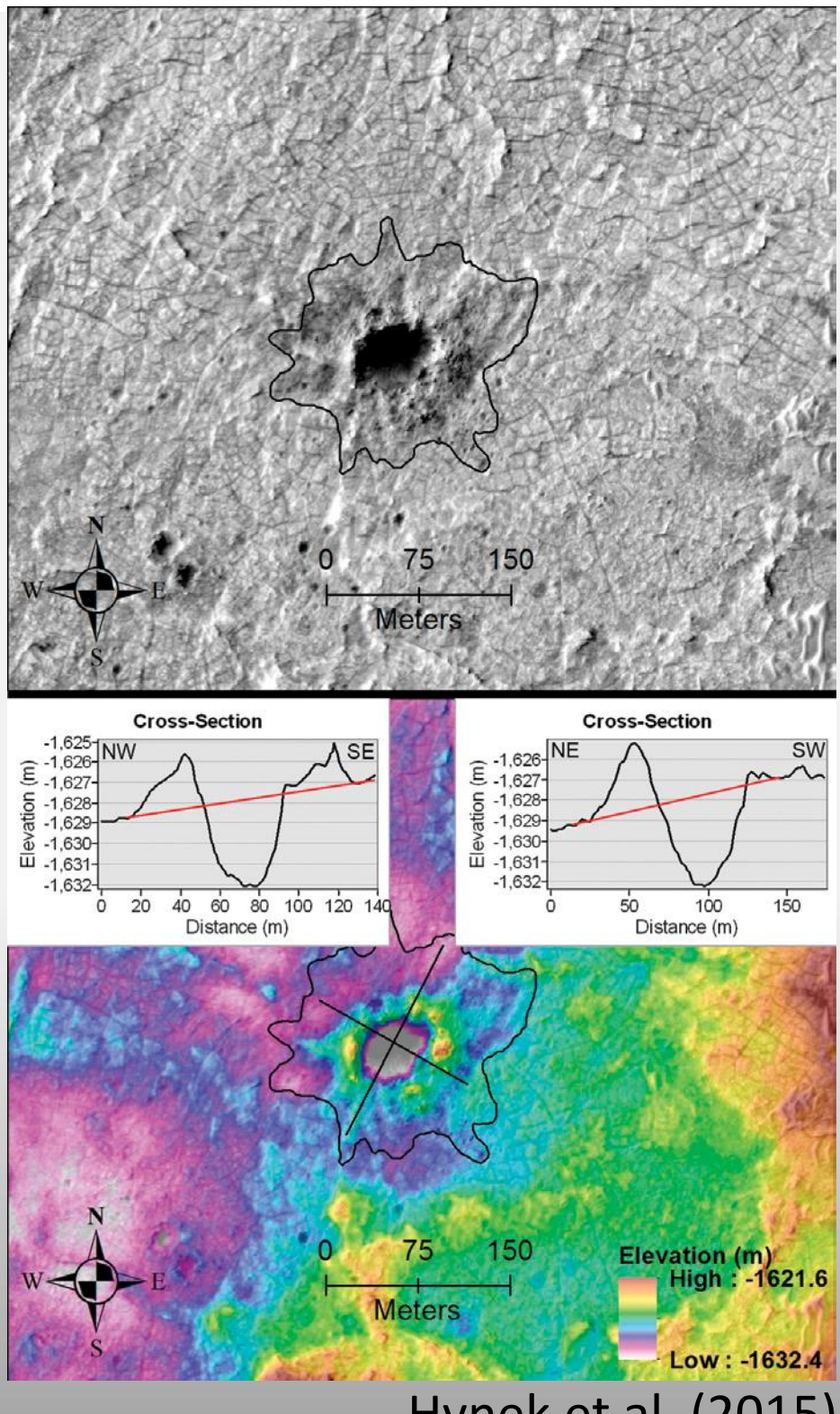

Hynek et al. (2015) 


\section{Chlorides are widespread}

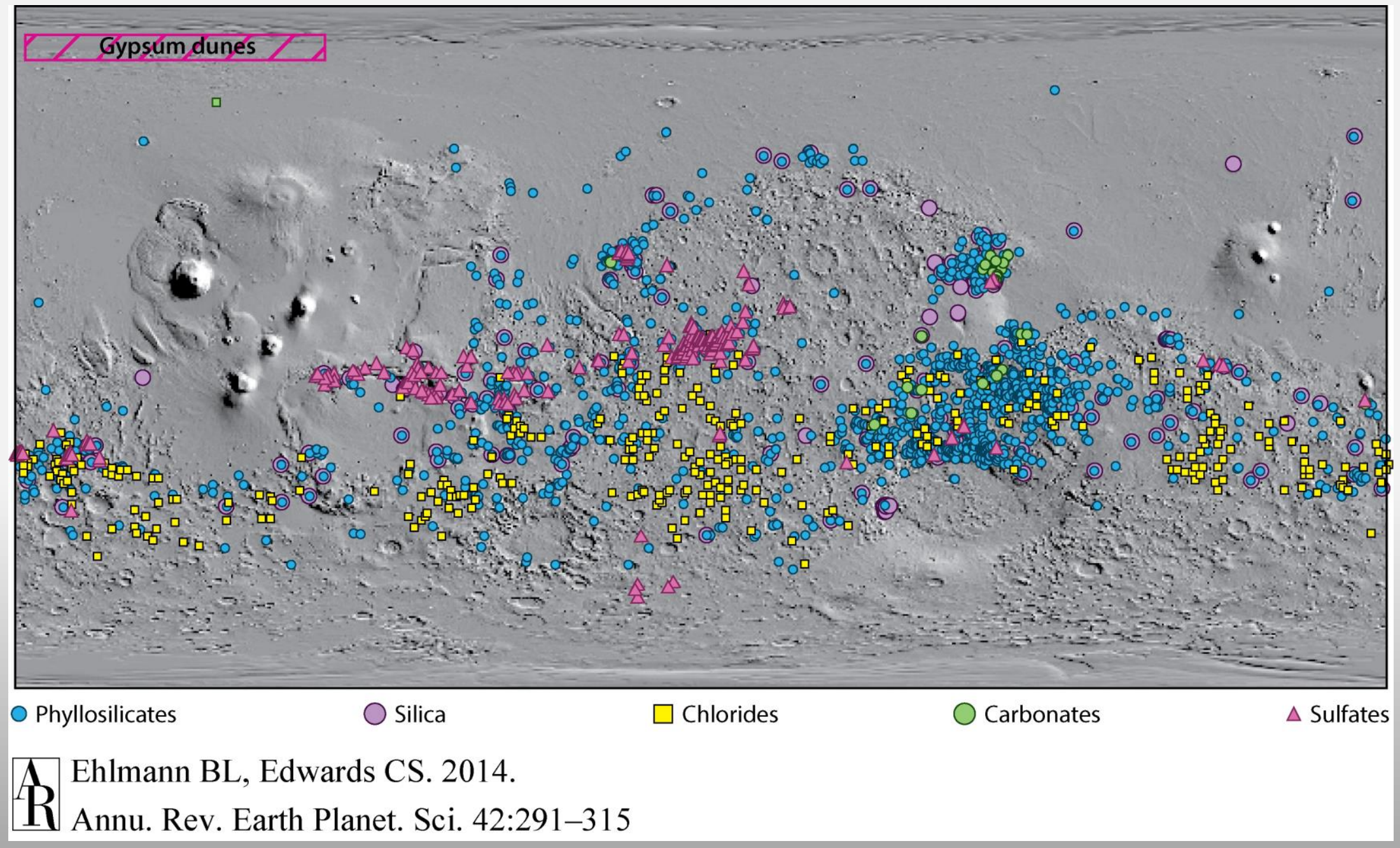




\section{Where did the $\mathrm{Cl}$ come from?}

A. Basaltic minerals (chlorapatite)

$\Rightarrow$ Weathered/leached by water

$\Rightarrow$ Discharged into lakes

B. Volcanic outgassing $(\mathrm{HCl})$

$\Rightarrow$ Aerosols

$\Rightarrow$ Dry deposition on surface $\left(\mathrm{ClO}_{4}^{-}{ }^{-}\right)$

$\Rightarrow$ "Washed" into lakes 


\section{Where did the $\mathrm{Cl}$ come from?}

A. Basaltic minerals (chlorapatite) $\mathrm{Cl}$ abundance (e.g. Filiberto +, 2016, M\&PS) $\Rightarrow$ Weathered/leached by water Martian breccia NWA 7034 (Santos +, 2015, GCA) $\Rightarrow$ Discharged into lakes

- Fast apatite weathering (e.g. Hurowitz \& McLennan, 2007, EPSL)

B. Volcanic outgassing $(\mathrm{HCl})$

$\Rightarrow$ Aerosols

$\Rightarrow$ Dry deposition on surface $\left(\mathrm{ClO}_{4}^{-}\right.$?)

$\Rightarrow$ "Washed" into lakes

Melwani Daswani \& Kite, in prep. 


\section{Where did the $\mathrm{Cl}$ come from?}

A. Basaltic minerals (chlorapatite) $\mathrm{Cl}$ abundance (e.g. Filiberto +, 2016, M\&PS) $\Rightarrow$ Weathered/leached by water Martian breccia NWA 7034 (Santos +, 2015, GCA) $\Rightarrow$ Discharged into lakes

- Fast apatite weathering (e.g. Hurowitz \& McLennan, 2007, EPSL)

Estimated (e.g. Craddock \&

B. Volcanic outgassing $(\mathrm{HCl})$ Greeley, 2009, Icarus) $\Rightarrow$ Aerosols

Formation (e.g. Smith+, 2014, (carus)

Phoenix WCL (Hecht +,

$\Rightarrow$ Dry deposition on surface $\left(\mathrm{ClO}_{4}^{-}{ }^{-}\right.$?) 2009, Science) $\Rightarrow$ "Washed" into lakes

Melwani Daswani \& Kite, in prep. 


\section{Weathering basalt}

Melwani Daswani \& Kite, in prep.

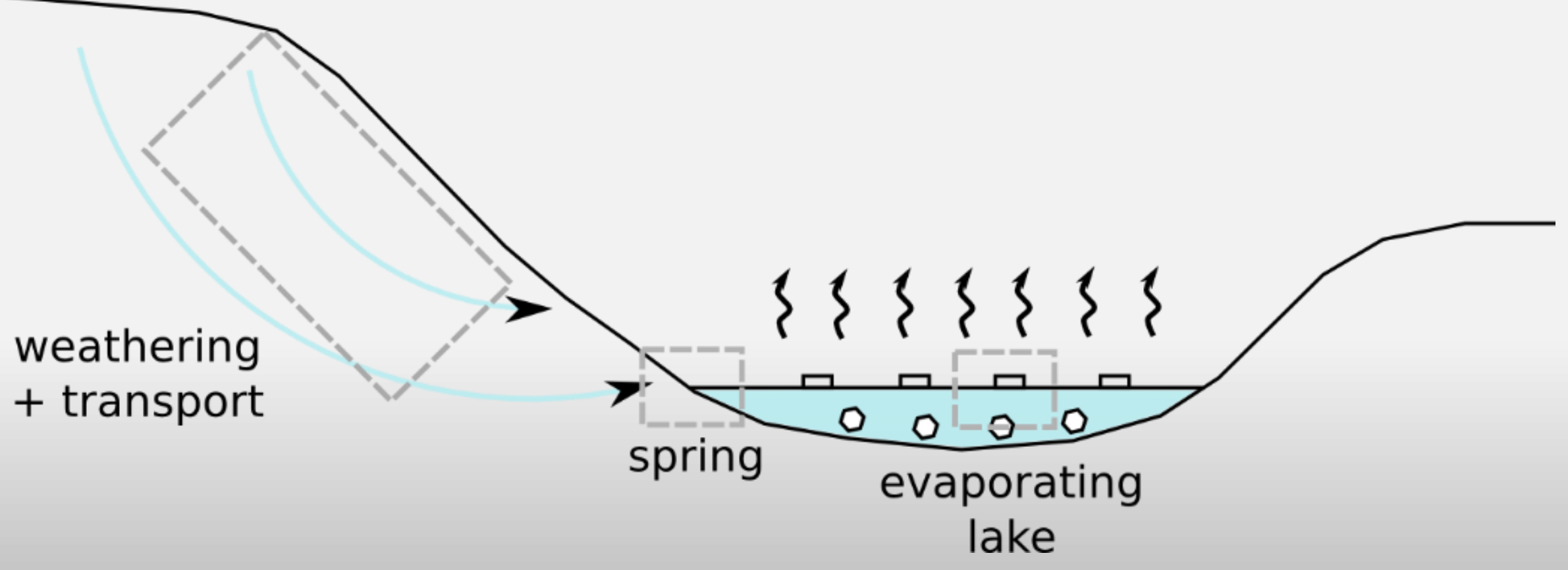




\section{Thermochemical modeling method}

Atmosphere

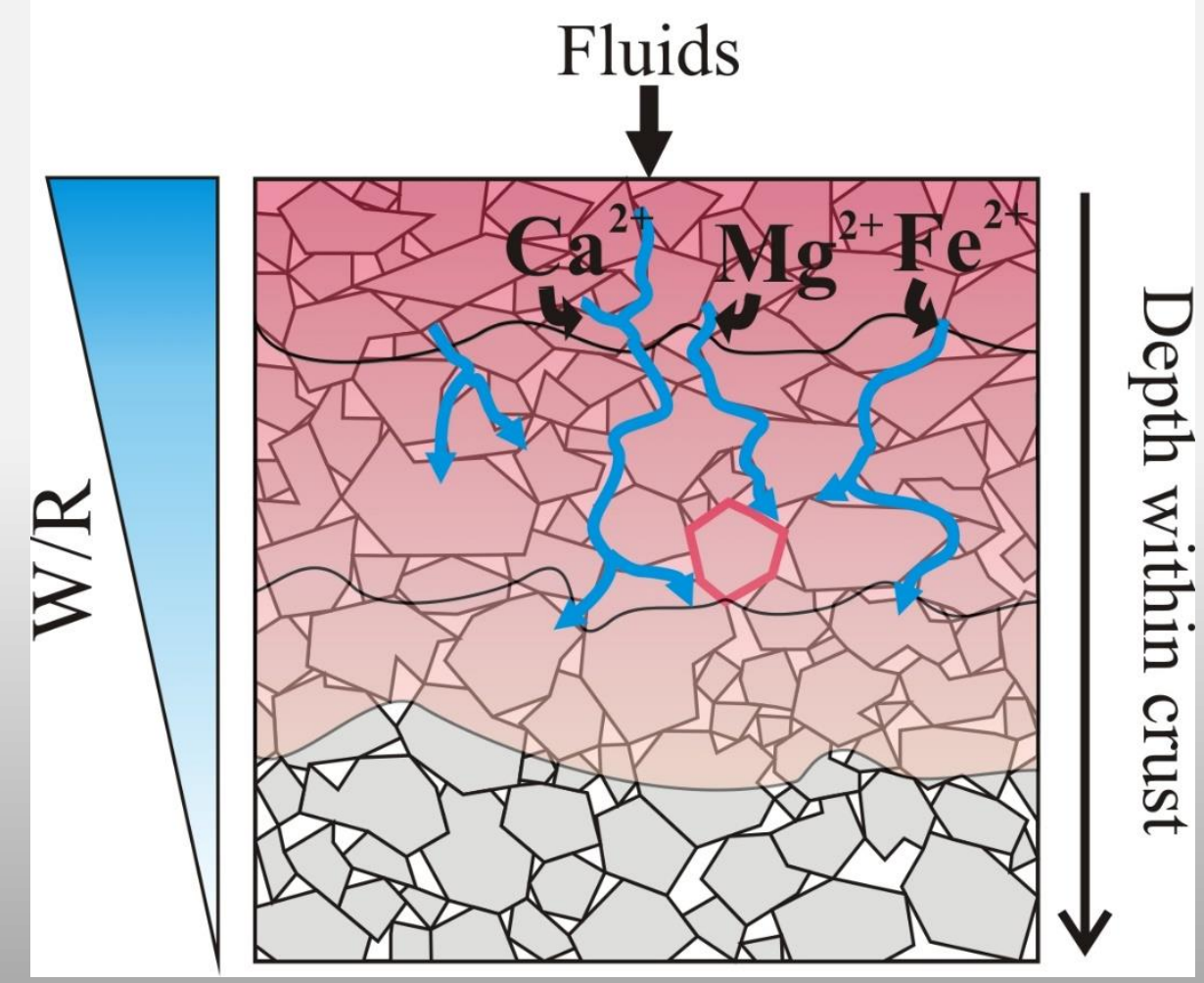

- CHIM-XPT code (Reed, 1998)

- Debye-Hückel theory

- Soltherm thermodynamic database (mostly derived from Holland \& Powell 2011 and ASU GEOPIG SUPCRT database)

Allows computations of:

- mineral stabilities and precipitation

- aqueous speciation

- mineral-gas-liquid equilibria

- enthalpies, P-T, pH, Eh 


\section{Result: minerals formed by weathering}

Incongruent dissolution, no apatite re-precipitation allowed

60 mbar initial $p \mathrm{CO}_{2}$

$0.01{ }^{\circ} \mathrm{C}$

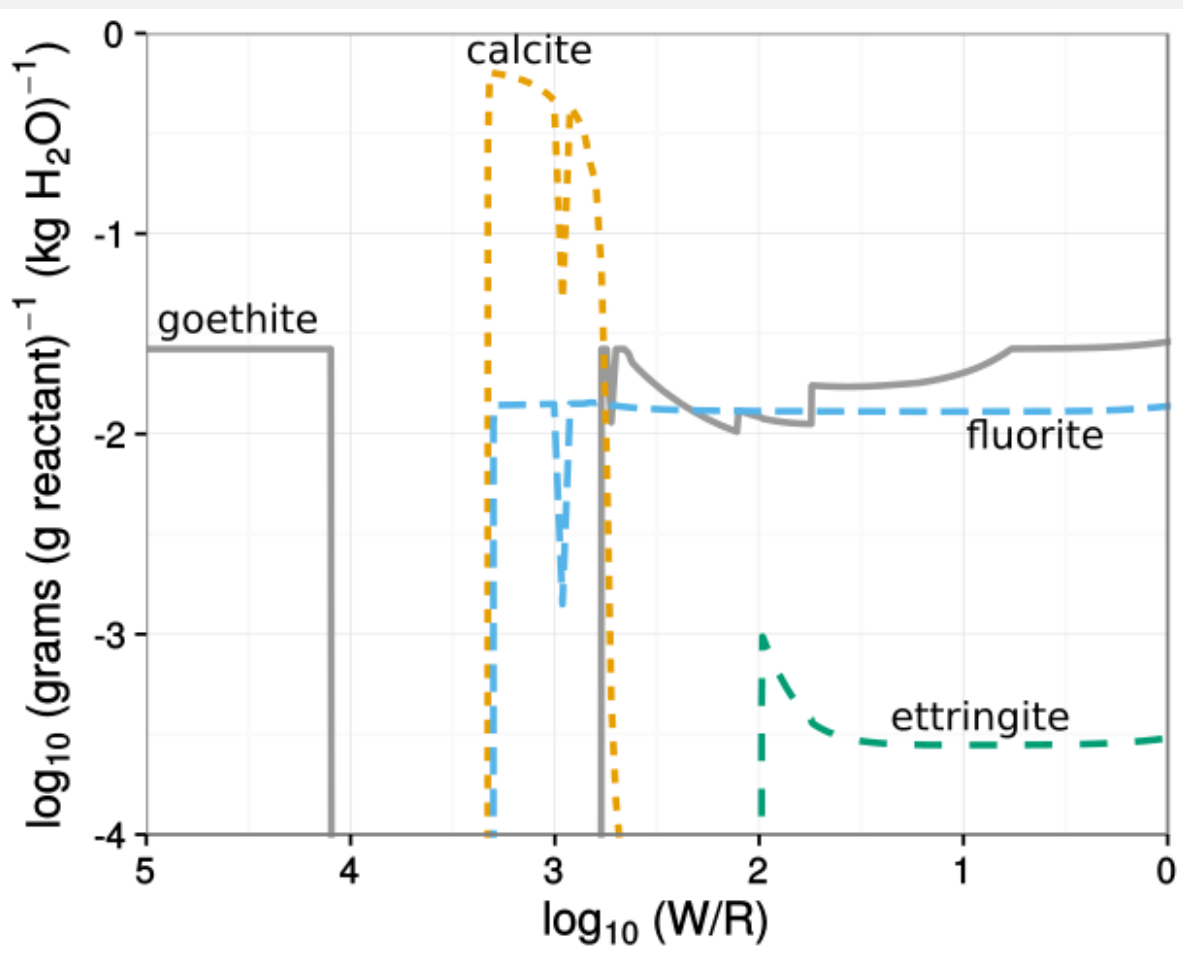

Melwani Daswani \& Kite, in prep.

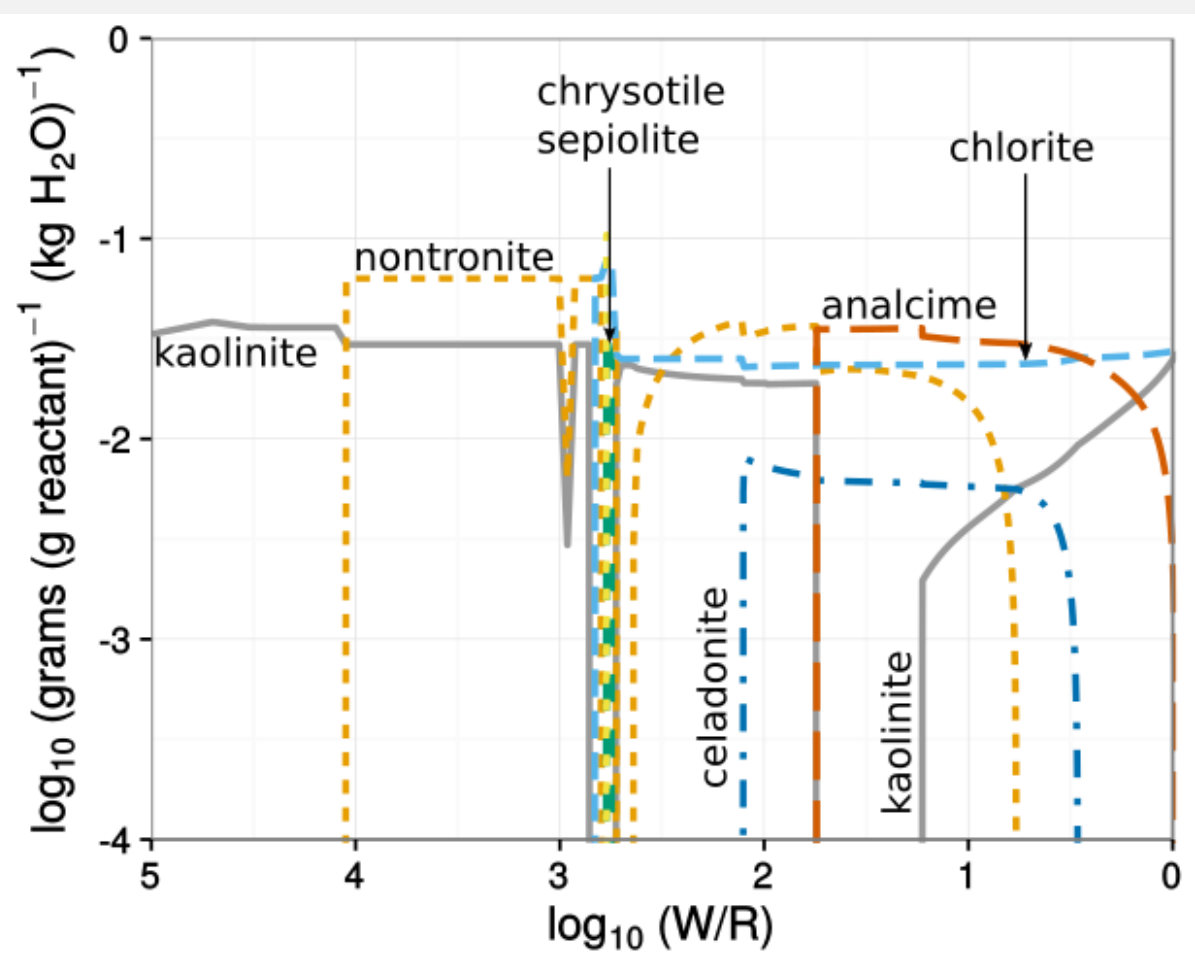




\section{Result: evolution of the fluid composition}

Incongruent dissolution, no apatite re-precipitation allowed

60 mbar initial $p \mathrm{CO}_{2}$

$0.01{ }^{\circ} \mathrm{C}$

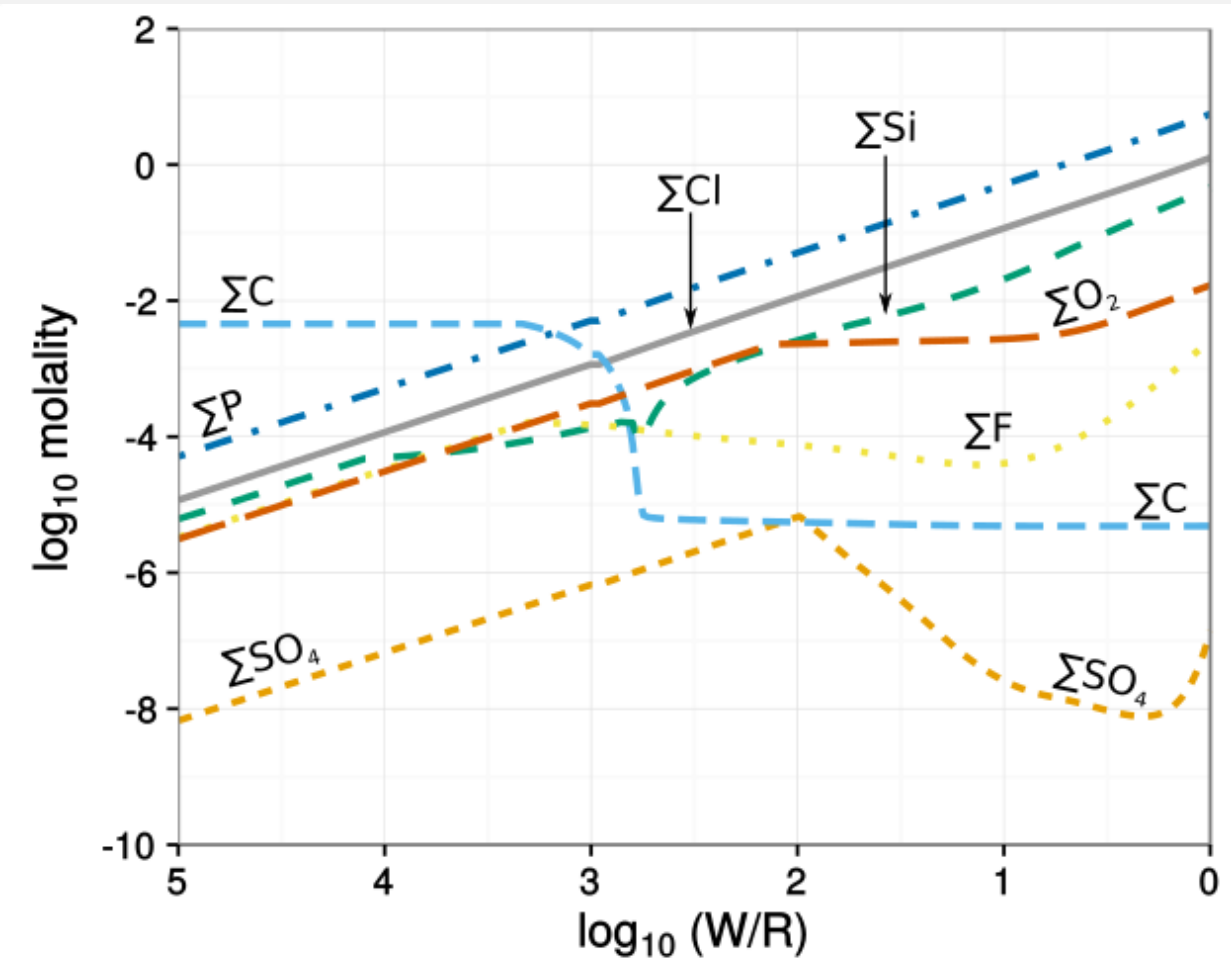

Melwani Daswani \& Kite, in prep.

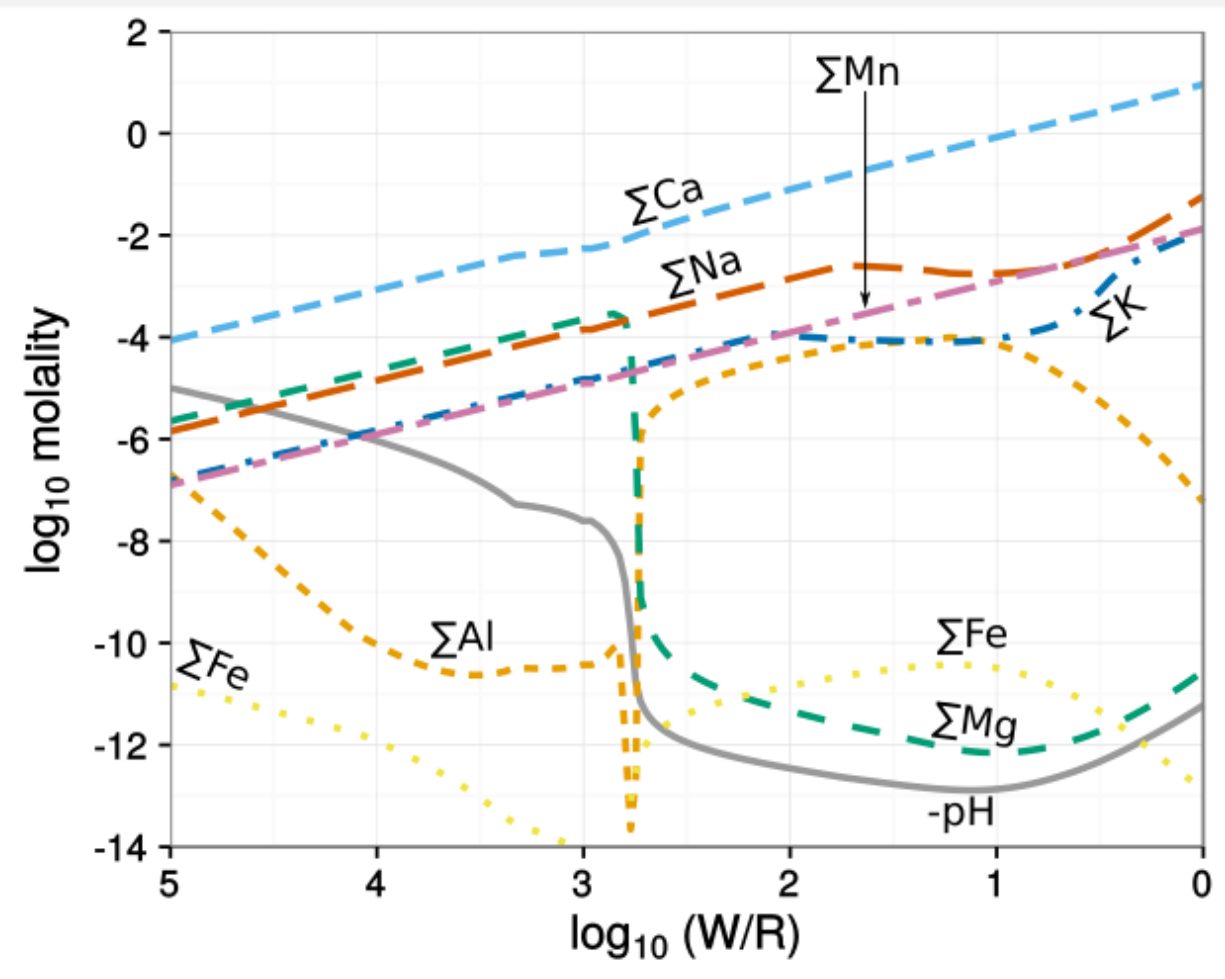




\section{Quantifying $\mathrm{H}_{2} \mathrm{O}$ and $\mathrm{Cl}$ required}

At $W / R=1$

$\Sigma \mathrm{Cl}=1.26 \mathrm{~mol} / \mathrm{kg} \mathrm{H}_{2} \mathrm{O}$

$=4.48 \times 10^{-2} \mathrm{~kg} \mathrm{Cl} / \mathrm{kg} \mathrm{H} \mathrm{H}_{2} \mathrm{O}$

Assuming all $\mathrm{Cl}$ in solution precipitates as $\mathrm{NaCl}$ in the basin $\left(\sim 36 \mathrm{~km}^{3}\right)$ we were looking at previously $\left(0.12 \mathrm{~km}^{3}\right.$ salt, $45 \%$ porosity): $8.6 \times 10^{10} \mathrm{~kg} \mathrm{Cl}$ :

$1.9 \times 10^{12} \mathrm{~kg} \mathrm{H}_{2} \mathrm{O}$ required

$=1.9 \times 10^{9} \mathrm{~m}^{3} \mathrm{H}_{2} \mathrm{O}$

$\approx 0.05$ times the volume of the basin

( $\approx 1.6 \mathrm{~m} \mathrm{H}_{2} \mathrm{O}$ across basin)
Melwani Daswani \& Kite, in prep.

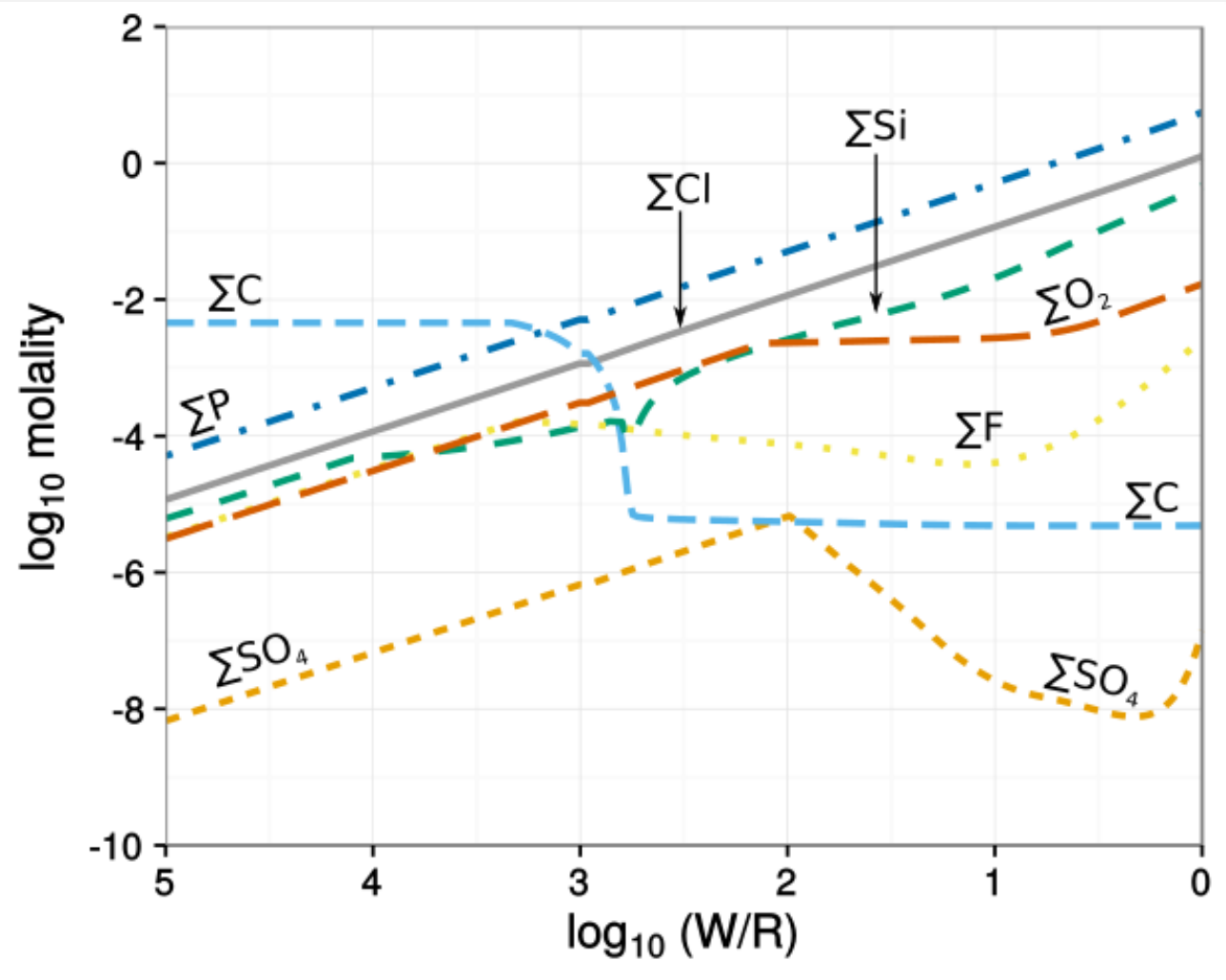




\section{Enough $\mathrm{Cl}$ from volcanic outgassing?}

Estimated (e.g. Craddock \&

Volcanic outgassing $(\mathrm{HCl})$ Greeley, 2009, Icarus) $\Rightarrow$ Aerosols

Formation (e.g. Smith+, 2014, Icarus)

Phoenix WCL (Hecht +,

$\Rightarrow$ Dry deposition on surface $\left(\mathrm{ClO}_{4}^{-}{ }^{-}\right.$?)

2009, Science)

$\Rightarrow$ "Washed" into lakes 


\section{Enough $\mathrm{Cl}$ from volcanic outgassing?}

\section{Estimated (e.g. Craddock \& \\ Volcanic outgassing $(\mathrm{HCl})$ \\ Greeley, 2009, Icarus) Aerosols}

Formation (e.g. Smith+, 2014, Icarus)

Phoenix WCL (Hecht +,

$\Rightarrow$ Dry deposition on surface $\left(\mathrm{ClO}_{4}^{-}\right.$?) 2009, Science)

Table 3

Table 1

Extrusive volumes $\left(10^{5} \mathrm{~km}^{3}\right)$ of martian volcanic material with time ${ }^{\mathrm{a}}$.

\begin{tabular}{lll}
\hline Epoch & $\begin{array}{l}\text { Extruded } \\
\text { volume }\end{array}$ & $\begin{array}{l}\text { Mass }^{\mathrm{b}} \\
\left(10^{22} \mathrm{~g}\right)\end{array}$ \\
\hline Late Amazonian & 2.11 & 0.7 \\
Middle Amazonian & 8.49 & 2.8 \\
Early Amazonian & 15.76 & 5.2 \\
Late Hesperian & 15.63 & 5.16 \\
\hline Early Hesperian & 17.65 & 5.82 \\
\hline Late Noachian & 7.77 & 2.56 \\
Middle Noachian & 1.39 & 0.46 \\
Early Noachian & $?$ & $?$ \\
Total & 68.8 & 22.7 \\
\hline
\end{tabular}

a Data from Greeley and Schneid (1991).

b Assumes a density of $3.3 \mathrm{~g} / \mathrm{cm}^{3}$.

Mass $\left(10^{18} \mathrm{~g}\right)$ of martian volcanic gases released through time.

\begin{tabular}{ll}
\hline Epoch & Constituents \\
\cline { 2 - 2 } & $\mathrm{HCl}$ \\
\hline Late Amazonian & 0.06 \\
Middle Amazonian & 0.22 \\
Early Amazonian & 0.42 \\
Late Hesperian & 0.41 \\
\hline Early Hesperian & 0.47 \\
\hline Late Noachian & 0.2 \\
Middle Noachian & 0.04 \\
Early Noachian & $?$ \\
Total & 1.82 \\
\hline
\end{tabular}

Adapted from Craddock \& Greeley (2009) 


\section{Enough $\mathrm{Cl}$ from volcanic outgassing?}

\section{Estimated (e.g. Craddock \& \\ Volcanic outgassing $(\mathrm{HCl})$ \\ Greeley, 2009, Icarus) Aerosols}

Formation (e.g. Smith+, 2014, Icarus)

Phoenix WCL (Hecht +,

$\Rightarrow$ Dry deposition on surface $\left(\mathrm{ClO}_{4}^{-}\right.$?) 2009, Science)

Table 3

Table 1

Extrusive volumes $\left(10^{5} \mathrm{~km}^{3}\right)$ of martian volcanic material with time ${ }^{\mathrm{a}}$.

\begin{tabular}{lll}
\hline Epoch & $\begin{array}{l}\text { Extruded } \\
\text { volume }\end{array}$ & $\begin{array}{l}\text { Mass }^{\mathrm{b}} \\
\left(10^{22} \mathrm{~g}\right)\end{array}$ \\
\hline Late Amazonian & 2.11 & 0.7 \\
Middle Amazonian & 8.49 & 2.8 \\
Early Amazonian & 15.76 & 5.2 \\
Late Hesperian & 15.63 & 5.16 \\
\hline Early Hesperian & 17.65 & 5.82 \\
\hline Late Noachian & 7.77 & 2.56 \\
Middle Noachian & 1.39 & 0.46 \\
Early Noachian & $?$ & $?$ \\
Total & 68.8 & 22.7 \\
\hline
\end{tabular}

a Data from Greeley and Schneid (1991).

b Assumes a density of $3.3 \mathrm{~g} / \mathrm{cm}^{3}$.

Mass $\left(10^{18} \mathrm{~g}\right)$ of martian volcanic gases released through time.

\begin{tabular}{ll}
\hline Epoch & Constituents \\
\cline { 2 - 2 } & \multicolumn{1}{c}{$\mathrm{HCl}$} \\
\hline Late Amazonian & 0.06 \\
Middle Amazonian & 0.22 \\
Early Amazonian & 0.42 \\
Late Hesperian & 0.41 \\
\hline Early Hesperian & $0.47 \approx 3.3 \mathrm{~kg} \mathrm{~m} \mathrm{~m}^{-2}$ \\
Late Noachian & 0.2 globally \\
Middle Noachian & 0.04 ? \\
Early Noachian & 1.82 \\
Total & \\
\hline
\end{tabular}

Adapted from Craddock \& Greeley (2009) 


\section{Detailed mapping to improve mass balance constraints}

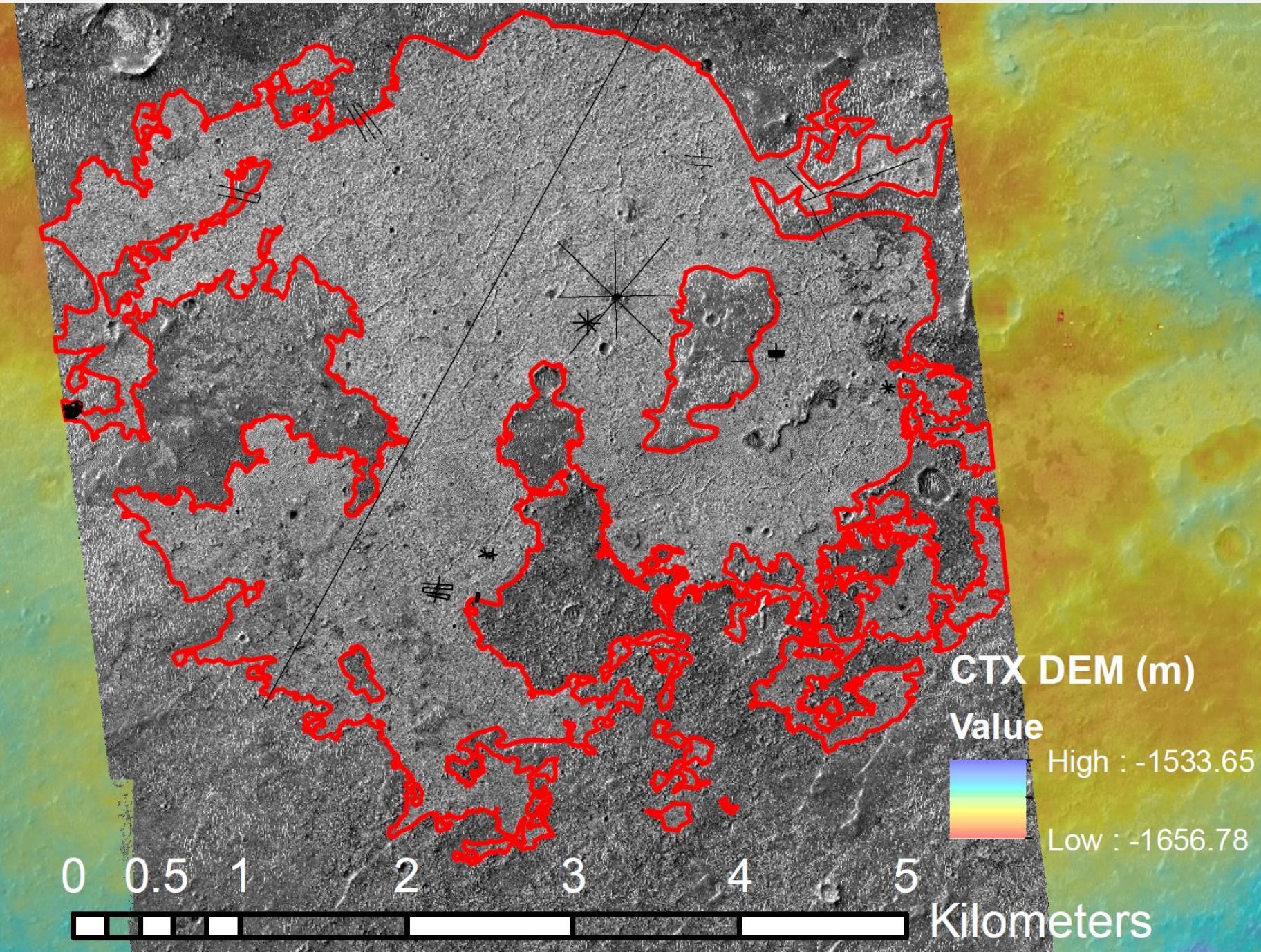

Melwani Daswani \& Kite, in prep.

Chloride deposit: Thickness: $1.5 \mathrm{~m}$ Volume: $\sim 2.15 \times 10^{7} \mathrm{~m}^{3}$

\begin{tabular}{|l|l|l|}
\hline & $\mathrm{NaCl}(\mathrm{kg})$ & $\mathrm{Cl}(\mathrm{kg})$ \\
\hline $\begin{array}{l}10 \% \\
\mathrm{NaCl}\end{array}$ & $4.7 \times 10^{9}$ & $2.8 \times 10^{9}$ \\
\hline $\begin{array}{l}25 \% \\
\mathrm{NaCl}\end{array}$ & $1.2 \times 10^{10}$ & $7.1 \times 10^{9}$ \\
\hline $\begin{array}{l}\text { Hynek et } \\
\text { al., 2015 }\end{array}$ & $1.4 \times 10^{11}$ & $8.5 \times 10^{10}$ \\
\hline
\end{tabular}

THE UNIVERSITY OF CHICAGO 


\section{$\mathrm{Cl}$ masses are somewhat different for other deposits}

\begin{tabular}{|c|c|c|c|c|}
\hline & $\begin{array}{l}\text { Near Miyamoto } \\
\text { Crater (this } \\
\text { study) }\end{array}$ & $\begin{array}{l}\text { Near Miyamoto } \\
\text { Crater (Hynek et } \\
\text { al. 2015) }\end{array}$ & $\begin{array}{l}\text { Terra Sirenum } \\
\text { (this study) }\end{array}$ & $\begin{array}{l}\text { West of Knobel } \\
\text { Crater (this } \\
\text { study) }\end{array}$ \\
\hline $\begin{array}{l}\text { Mean deposit } \\
\text { thickness (m) }\end{array}$ & 1.5 & 4 & 2.6 & 8.0 \\
\hline Basin area $\left(\mathrm{m}^{2}\right)$ & $8.4 \times 10^{8}$ & $1.2 \times 10^{9}$ & $3.5 \times 10^{9}$ & $3.5 \times 10^{9}$ \\
\hline $\begin{array}{l}\text { Deposit volume } \\
\left(\mathrm{m}^{3}\right)\end{array}$ & $2.2 \times 10^{7}$ & $1.2 \times 10^{8}$ & $5.8 \times 10^{7}$ & $4.2 \times 10^{6}$ \\
\hline $\begin{array}{l}10 \% \mathrm{NaCl} \text { mass } \\
\left(\mathrm{Cl} \mathrm{kg} / \mathrm{m}^{2}\right)\end{array}$ & 3.4 & 12.8 & 2.2 & 0.2 \\
\hline $\begin{array}{l}25 \% \mathrm{NaCl} \text { mass } \\
\left(\mathrm{Cl} \mathrm{kg} / \mathrm{m}^{2}\right)\end{array}$ & 8.5 & 32 & 5.5 & 0.4 \\
\hline
\end{tabular}




\section{Summary/conclusions}

\section{Origin of the $\mathrm{Cl}$}

Near-subsurface basalt weathering

+ Can "hide" sulfates in subsurface

+ Can make clay minerals prior to chlorides

+ Mass balance consistent

- Requires $>1$ season of T above freezing

\section{Volcanic $\mathrm{Cl}$ phases}

+ Cl-phases detected on the surface, probably volcanogenic

+ Mass balance consistent

+ Does not require subsurface fluids

+ Consistent with inverted channels

- Sulfur is transported to lake 



\section{Fluid inclusions in chlorides can preserve DNA, microorganisms.}
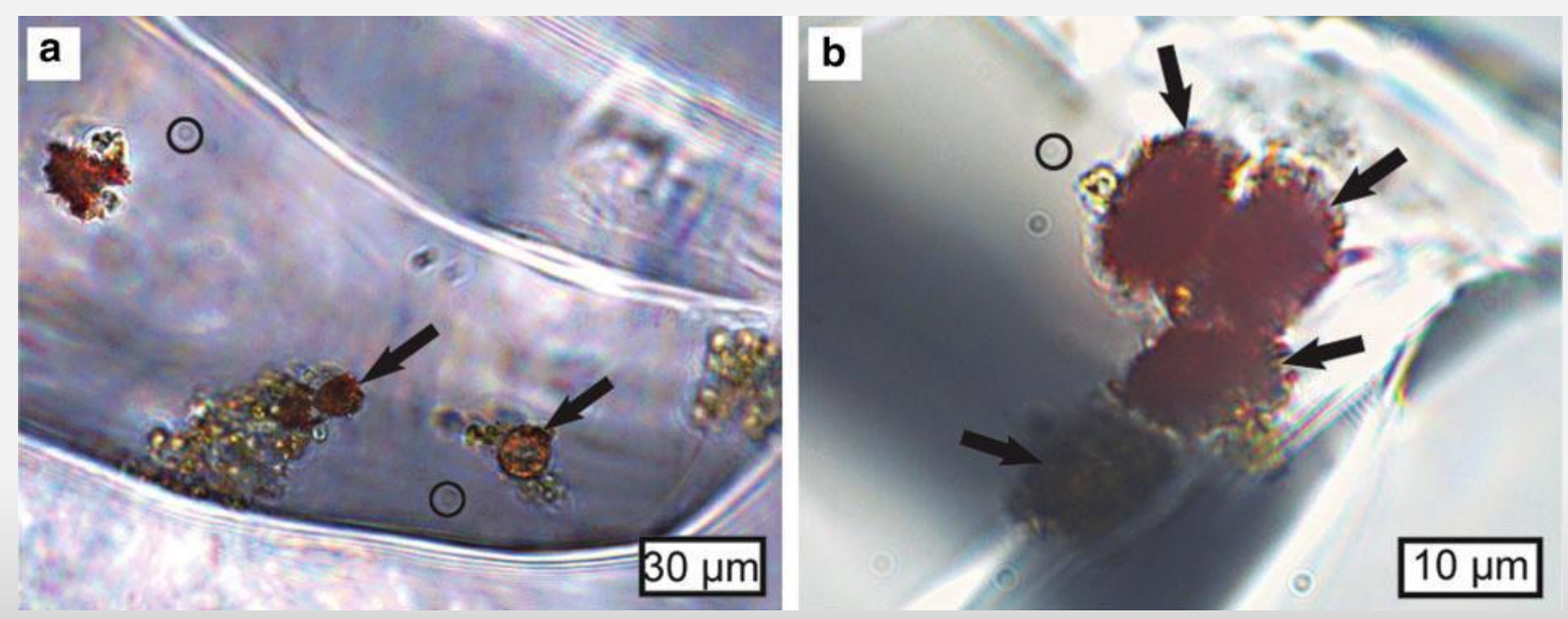

Photomicrographs of single-celled algae and prokaryotes in fluid inclusions in halite from Qaidam Basin (Wang et al. 2016, Astrobiology) 


\section{Fluid inclusions in chlorides can preserve DNA, microorganisms.}
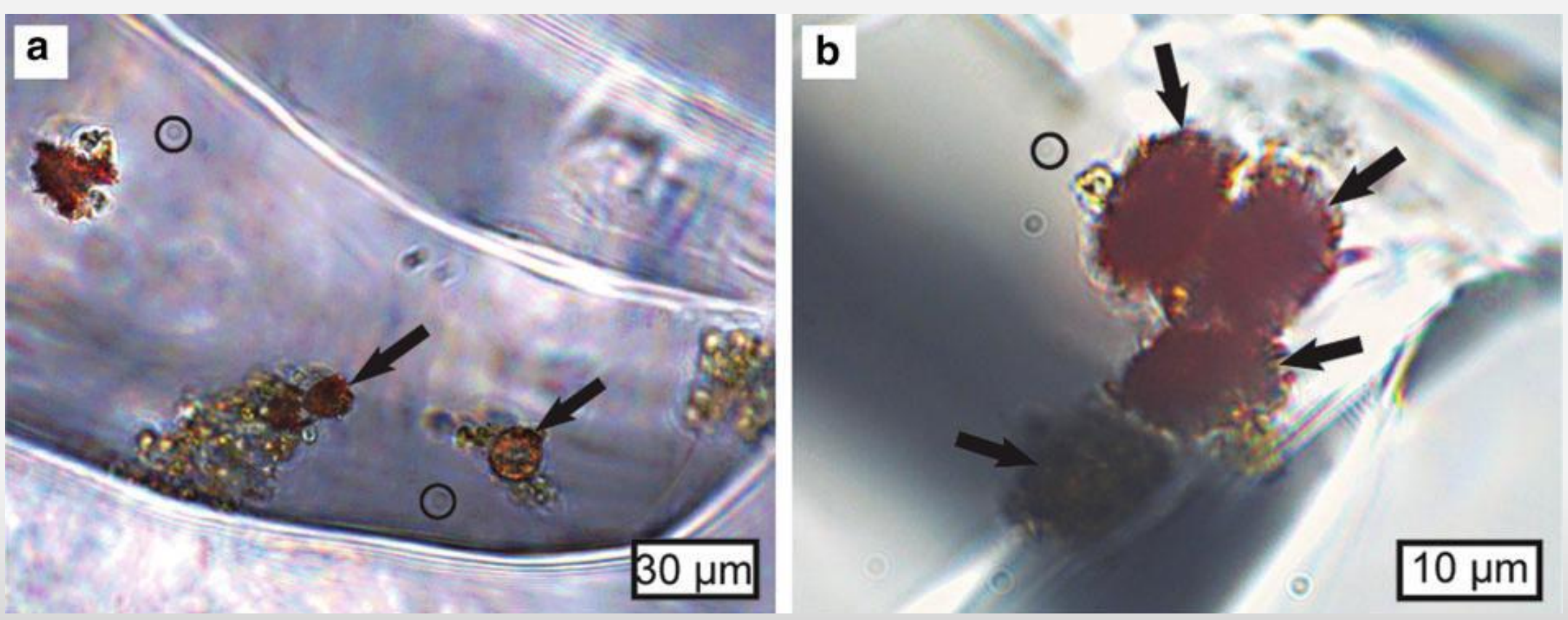

Photomicrographs of single-celled algae and prokaryotes in fluid inclusions in halite from Qaidam Basin (Wang et al. 2016, Astrobiology)

\section{Possible shielding from GCR damage?}




\section{Chlorides are widespread}

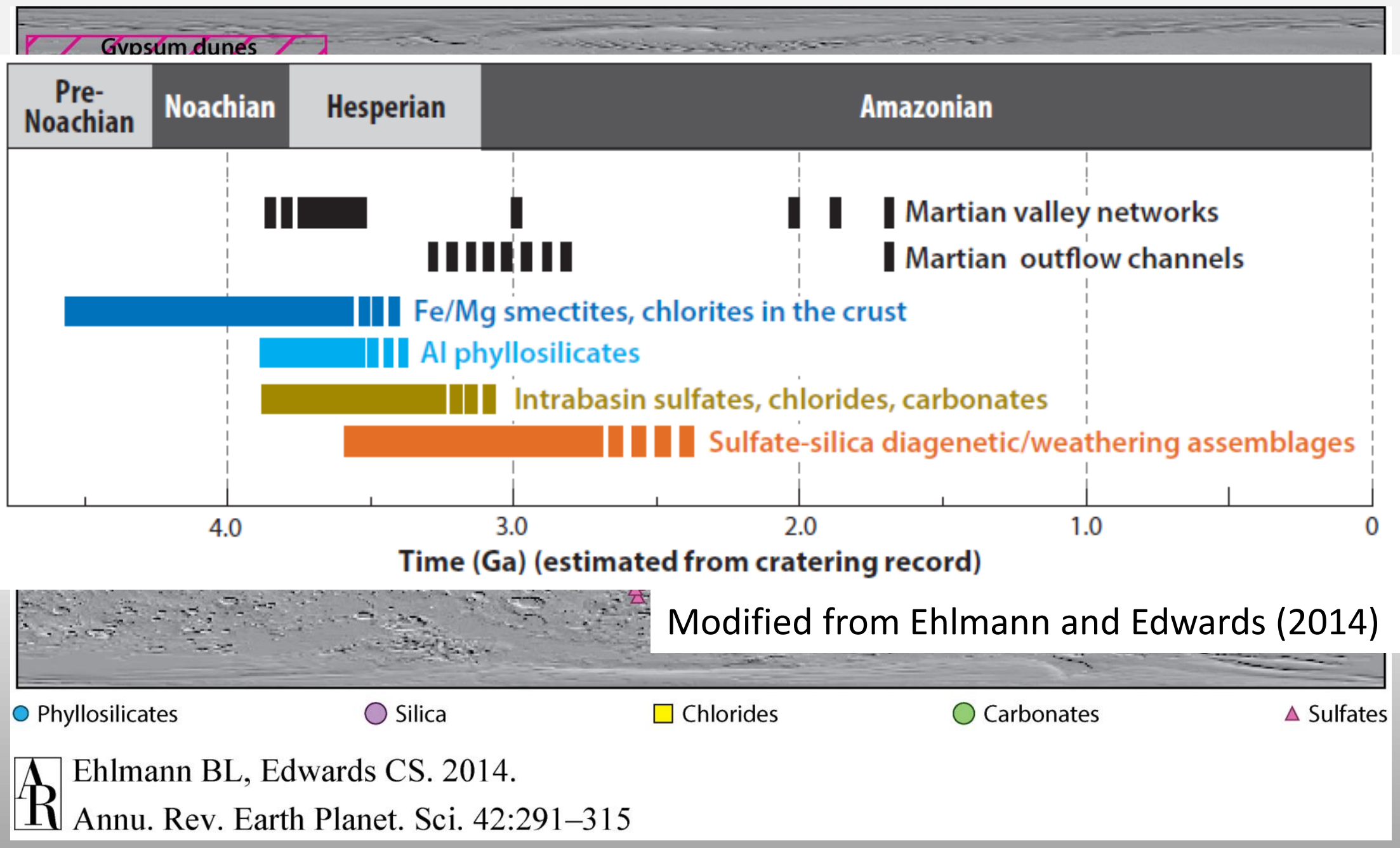

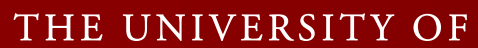

CHICAGO 


\section{Finding a representative basalt composition}

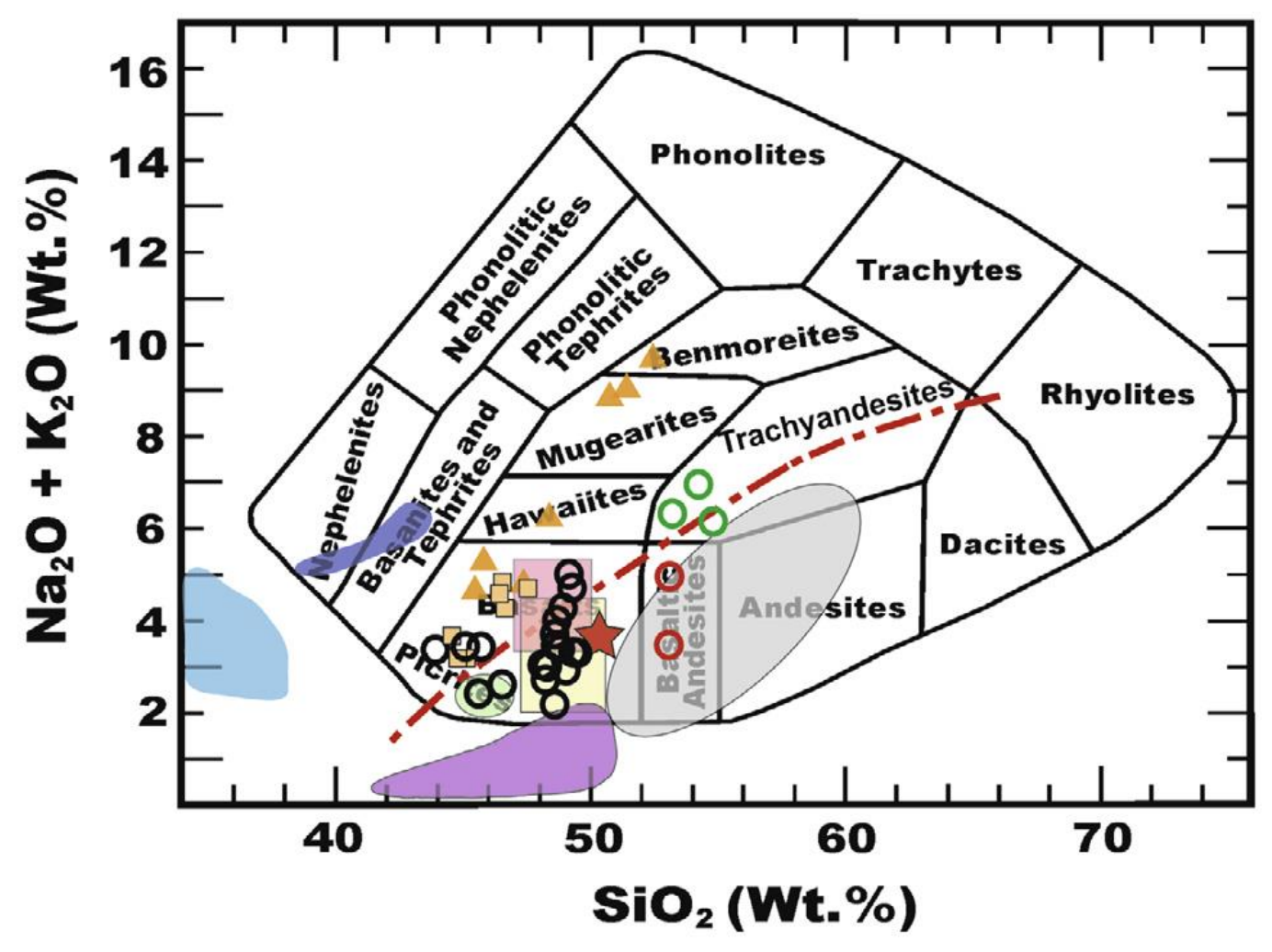

O Basalt Clasts

O Basaltic Andesite Clasts

Trachyandesite Clasts FTP Clast Estimate

NWA 7034 Bulk Matrix
A Gale Crater Rocks $\square$ GRS Global

MER A-class $\longrightarrow$ SNC TES Global

Wishstone and Champagne Microbasalt and CLIMR
NWA 7034 basalt clasts

\begin{tabular}{|l|l|l|}
\hline Mineral & $\begin{array}{l}\text { Mode } \\
\text { Vol. \% }\end{array}$ & $\begin{array}{l}\text { Norm. wt. } \\
\%\end{array}$ \\
\hline Plag. & 50 & 45.8 \\
\hline K-felds & 2 & 1.8 \\
\hline $\begin{array}{l}\text { Low Ca } \\
\text { pyx }\end{array}$ & 20 & 24.1 \\
\hline
\end{tabular}

High Ca 10

pyx

\begin{tabular}{l|l|l} 
Apatite & 5 & 6.0
\end{tabular}

$\begin{array}{lll}\text { Magnetite } & 3 & 5.4\end{array}$

\begin{tabular}{l|l|l} 
Ilmenite & 1 & 4.6
\end{tabular}

Apatite contains $\sim 4.6$ wt. $\% \mathrm{Cl}$

Santos et al. (2015), Geochim. Cosmochim. Acta 157, $56-85$. 


\section{Minerals weather at different rates}

$A_{1}$

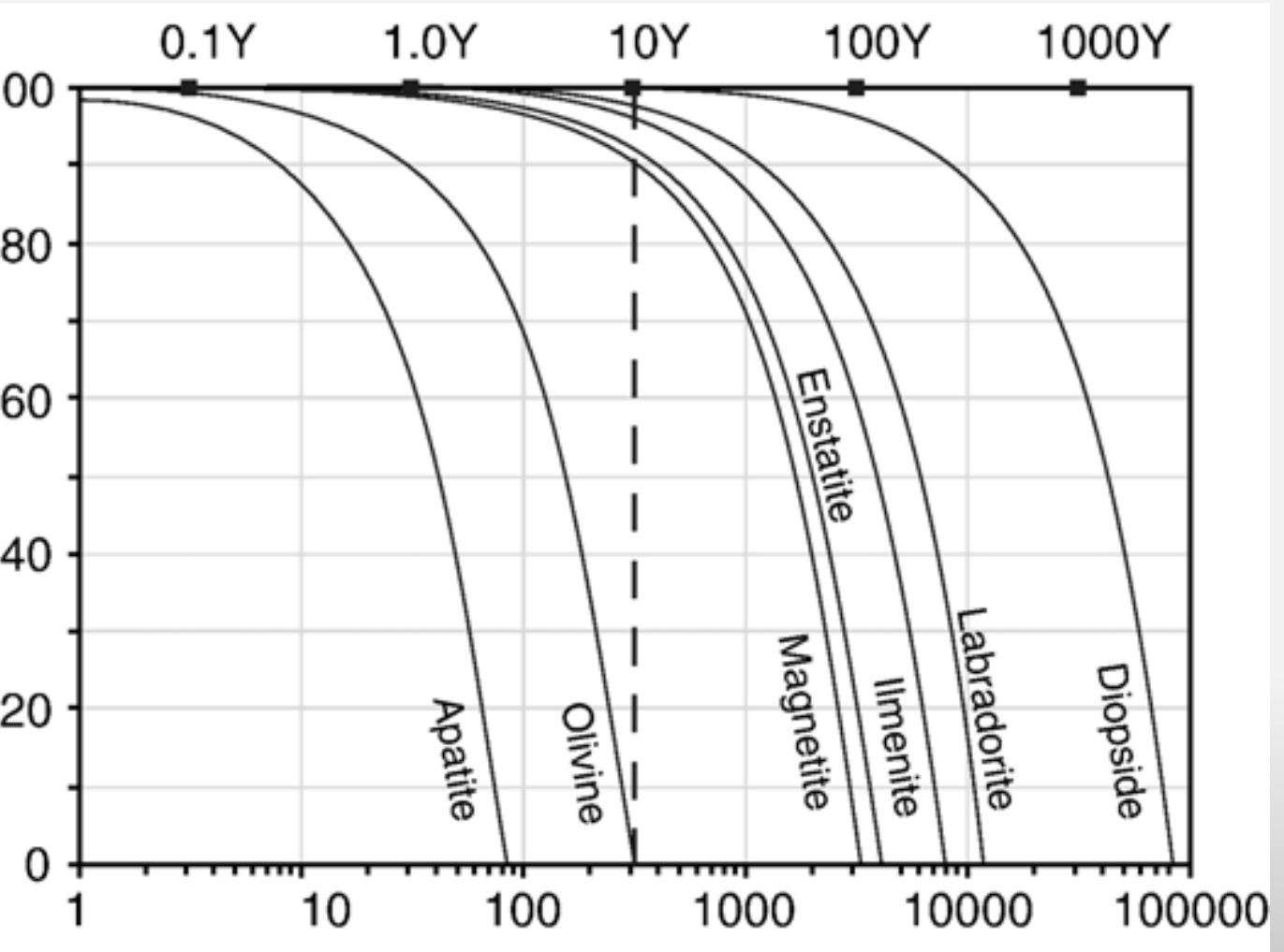

Water/Rock

Adjusted reactant rock composition for 90 wt. \% apatite

(Melwani Daswani \& Kite, in prep.)

\begin{tabular}{|l|l|}
\hline Mineral & Wt .\% \\
\hline Plagioclase & 4.9 \\
\hline K-Felds & 0.2 \\
\hline Low Ca pyx & 2.6 \\
\hline High Ca pyx & 1.3 \\
\hline Apatite & 90.0 \\
\hline Magnetite & 0.6 \\
\hline Ilmenite & 0.5 \\
\hline Pyrite* & $0.004^{*}$ \\
\hline
\end{tabular}

From Hurowitz and McLennan (2007),

Earth Planet. Sci. Lett., 260, 432 - 443.

*Added and adjusted from Wittmann et al., 2015 M\&PS 50, 326 - 352. 


\section{Quantifying $\mathrm{H}_{2} \mathrm{O}$ and $\mathrm{Cl}$ required}

At $W / R=100$

$\Sigma \mathrm{Cl}=1.15 \times 10^{-2} \mathrm{~mol} / \mathrm{kg} \mathrm{H}_{2} \mathrm{O}$

$=4.1 \times 10^{-4} \mathrm{~kg} \mathrm{Cl} / \mathrm{kg} \mathrm{H}_{2} \mathrm{O}$

Assuming all $\mathrm{Cl}$ in solution precipitates as $\mathrm{NaCl}$ in the basin $\left(\sim 36 \mathrm{~km}^{3}\right)$ we were looking at previously $\left(0.12 \mathrm{~km}^{3}\right.$ salt, $45 \%$ porosity): $8.6 \times 10^{10} \mathrm{~kg} \mathrm{Cl}$ :

$2.1 \times 10^{14} \mathrm{~kg} \mathrm{H}_{2} \mathrm{O}$ required

$=2.1 \times 10^{11} \mathrm{~m}^{3} \mathrm{H}_{2} \mathrm{O}$

$\approx 5.9$ times the volume of the basin

( $\approx 172 \mathrm{~m} \mathrm{H}_{2} \mathrm{O}$ across basin)
Melwani Daswani \& Kite, in prep.

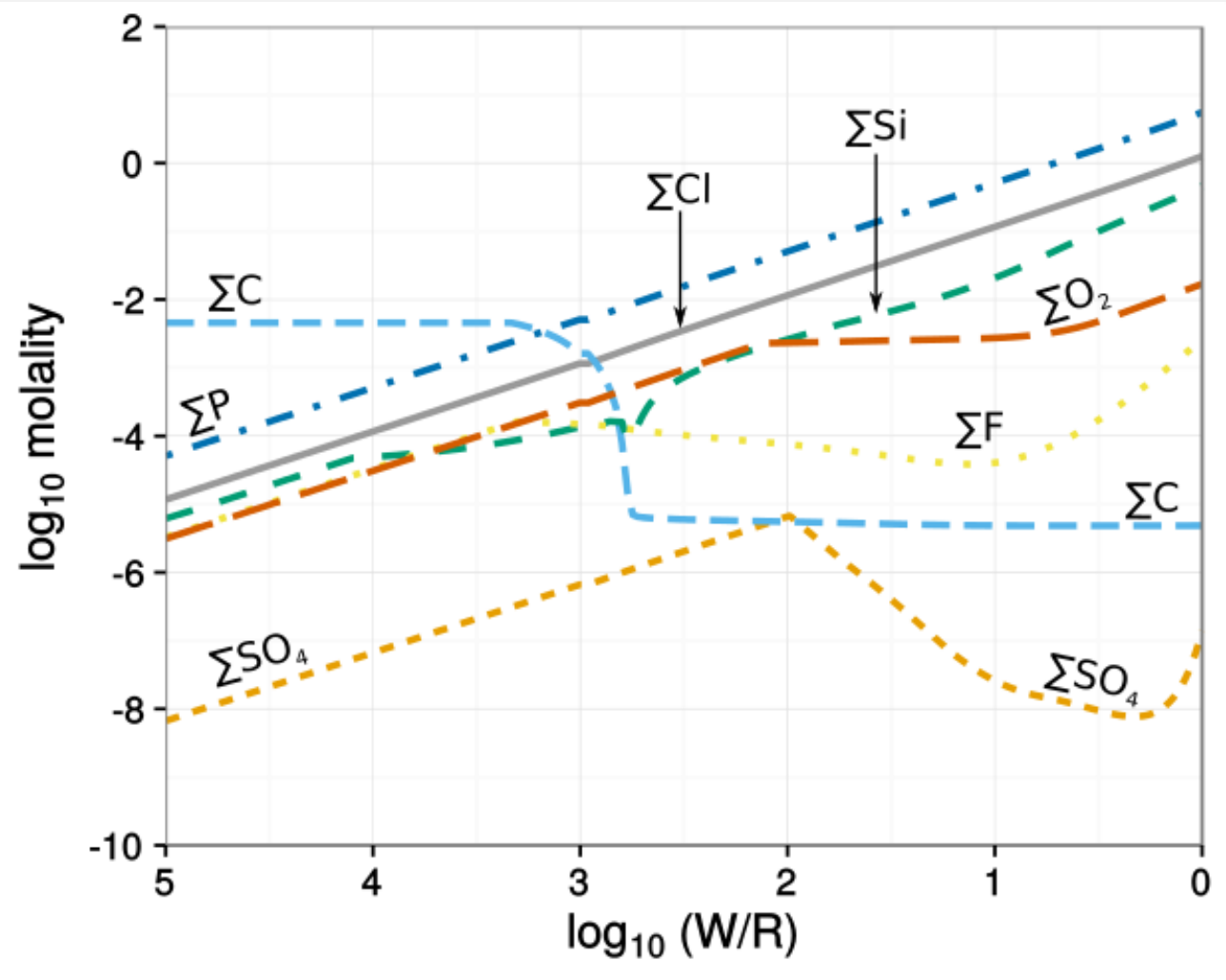




\section{What was the time scale of the event?}

We know the mass of the chloride deposit $\left(\sim 1.4 \times 10^{11} \mathrm{~kg}\right)$

and the surface area of the basin $\left(\sim 1.2 \times 10^{9} \mathrm{~m}^{2}\right)$ (Hynek et al., 2015)

Assumptions:

- Chlorides are $\mathrm{NaCl}$

- Porosity of the deposit is $45 \%$

- Diffusivity $(K)$ of the basin rock $=7 \times 10^{-7} \mathrm{~m}^{2} \mathrm{~s}^{-1}$ (typical for silicates)

We calculate $\sim 2.5 \times 10^{4} \mathrm{~kg}$ rock $/ \mathrm{m}^{2}$ (i.e. $~ 14.9 \mathrm{~m}$ depth) weathering

$$
\begin{gathered}
L=2.32 \sqrt{K \tau} \\
\tau \approx 6 \times 10^{7} \mathrm{~s} \approx 1 \text { Mars year }
\end{gathered}
$$

Melwani Daswani \& Kite, in prep. 


\section{Where did the $\mathrm{Cl}$ come from?}

C. Other sources?

Meteoritic? Cometary?

Ancient reworked deposits? 